\title{
OPEN Vacancy tuned thermoelectric properties and high spin filtering performance in graphene/silicene heterostructures
}

\author{
Zainab Gholami \& Farhad Khoeini ${ }^{\bowtie}$
}

The main contribution of this paper is to study the spin caloritronic effects in defected graphene/ silicene nanoribbon (GSNR) junctions. Each step-like GSNR is subjected to the ferromagnetic exchange and local external electric fields, and their responses are determined using the nonequilibrium Green's function (NEGF) approach. To further study the thermoelectric (TE) properties of the GSNRs, three defect arrangements of divacancies (DVs) are also considered for a larger system, and their responses are re-evaluated. The results demonstrate that the defected GSNRs with the DVs can provide an almost perfect thermal spin filtering effect (SFE), and spin switching. A negative differential thermoelectric resistance (NDTR) effect and high spin polarization efficiency (SPE) larger than $99.99 \%$ are obtained. The system with the DV defects can show a large spin-dependent Seebeck coefficient, equal to $S_{s} \sim 1.2 \mathrm{mV} / \mathrm{K}$, which is relatively large and acceptable. Appropriate thermal and electronic properties of the GSNRs can also be obtained by tuning up the DV orientation in the device region. Accordingly, the step-like GSNRs can be employed to produce high efficiency spin caloritronic devices with various features in practical applications.

The next generation of electronic devices is often required to possess high performance, minimum waste heat, and low dimension in practical applications ${ }^{1-4}$. The spin caloritronics, as a new branch of spintronics, focuses on the relationship between the heat and spin transports in the material and attempts to pave the way for the development of nanostructure devices in the heat sensors, waste heat recyclers, and future device technologies ${ }^{5-7}$. The spin-dependent Seebeck effect (SDSE), as an essential feature of the spin caloritronics, aims to produce spinpolarized currents by using the temperature gradient. This can efficiently help us to obtain a pure spin current with no charge companions. The spin equivalent of the classical Seebeck effect was first observed by Uchida et al. ${ }^{8}$ in the ferromagnetic metals. Numerous studies were then performed by other researchers on quasi-one or two-dimensional (2D) materials such as graphene (GE), silicene (SE), $\mathrm{MoS}_{2}$, and so on ${ }^{9-12}$. Although nanoribbon materials with zigzag edges have inherent antiferromagnetic properties, they can be magnetized by using an external magnetic field. This can provide us to understand well SDSE and thermal spin transport attributes of such types of materials (e.g., spin-dependent Seebeck diode (SSD) effect, thermal SFE, and thermal spin-giantmagnetoresistance (GMR) effect ${ }^{13-15}$ ).

GE and SE are now introduced as the famous family of low-dimensional materials ${ }^{16-18}$ with unusual features for the next generation of device applications ${ }^{19,20}$. They consist of a honeycomb structure with promising electronic properties such as high carrier mobility ${ }^{21-24}$. GE and SE nanostructures are now identified as materials with adequate Seebeck coefficient and widespread spin caloritronics applications ${ }^{21,23-25}$. Quasi-one-dimensional structures, theoretically, show satisfactory thermoelectric performance compared to 2D structures ${ }^{26}$. This may be attributed to the fact that cutting a 2D material in to a nanoribbon can decrease the thermal conductivity and lead to better thermoelectric properties. A GE-based thermoelectric device was experimentally introduced by Sierra et al. ${ }^{27}$, to produce a spin voltage via two ferromagnetic thermal sources with variant temperatures. However, GE is not often identified as an efficient thermoelectric material, because it shows a low figure of merit (FOM $)^{28}$. In contrast, SE has superior thermoelectric properties than GE and can drastically enhance the Seebeck coefficient, due to its nonzero band gap ${ }^{24,29}$. According to previous studies, the zigzag SE nanoribbons (ZSNRs) can suitably provide a significant spin filter efficiency ${ }^{30}$, and a perfect $\operatorname{SDSE}^{9}$. The thermoelectric performance of nanostructures can also be enhanced by various ways, such as hybridization ${ }^{31,32}$, defects $^{33-35}$, and doping ${ }^{36,37}$. As shown in the previous studies ${ }^{4,38,39}$, the hybrid nanostructures theoretically show superior thermoelectric properties than 
the similar materials with the single nanostructures. Several studies have already been performed to investigate various properties of the GSNRs in which GE and SE have vertically or laterally bonded together ${ }^{40-43}$. The results showed that the combination of GE with other 2D materials could lead to a hybrid structure with various properties and applications ${ }^{44-47}$. Nevertheless, there are little data about the electronic behavior, spin transport, and thermoelectric attributes of the GSNRs ${ }^{38,46}$, and further research is still required in this area.

Several studies have been conducted to investigate the effect of structural defects and pattern geometry on the thermal and thermoelectric phenomena of nanoribbons ${ }^{10,48-50}$. Experimental results clearly show that the thermal conductivity of GE with a patterned vacancy (nanomesh) structure is significantly lower than that observed by the pristine $\mathrm{GE}^{51}$. In addition, nanoribbons with periodic edge defects, called saw tooth-like (ST) ${ }^{52}$, can also improve the spin thermoelectric properties, identified by the large values of spin-dependent Seebeck coefficient and $\mathrm{FOM}^{53}$. Some designs have been used to connect the GE flake to two armchair GE nanoribbons (AGNRs), and to generate spin-dependent currents ${ }^{54,55}$. The width of the nanoribbon, the coupling between the leads and the ribbon, and the topology were also identified as the main factors affecting the electrical and thermal properties of the nanoribbon. Sonvane et al. showed that the thermal transport of a GNR could significantly be affected by changing its length or width ${ }^{56}$. Some unusual thermal and electrical transport properties have been observed in the asymmetric shapes of nanoribbons ${ }^{57-60}$. All these observations may be related to the symmetric or asymmetric connection of the device to the leads and quantum interference ${ }^{61}$. The thermal and thermoelectric properties of the GNRs have been studied by Rojo et al. ${ }^{62}$, and Tan et al. ${ }^{10}$, for the vertical and parallel step-like junctions, respectively. However, there is still little information about the thermoelectric phenomena of hybrid junctions with different geometry and structural defects. This is especially true for the parallel step-like GSNRs, inspiring us to explore the thermal transport attributes in this type of hybrid nanostructures.

In this study, the spin caloritronic effects in the parallel step-like graphene/silicene nanoribbon (GSNR) junctions, including the divacancy, are numerically studied using the tight-binding (TB) and NEGF approaches. Three GSNRs with different length-to-width ratios are first selected and then subjected to the ferromagnetic exchange and local external electric fields. There are many experimental studies available in the literature, which have focused on developing various nanostructures ${ }^{40,63-69}$, and measuring their spin thermoelectric properties ${ }^{70,71}$. However, this paper numerically investigates the thermoelectric properties of parallel step-like GSNRs because: (1) the nanoribbons can satisfactorily show thermoelectric performance compared to their corresponding 2D structures $^{72}(2)$ the step-like nanostructures can provide some unusual thermal and electrical transport properties for the asymmetric shape of nanoribbons ${ }^{62}(3)$ the hybrid type of nanostructures can often provide superior thermoelectric properties compared to the similar materials with the single nanostructures ${ }^{32}$, and (4) the defected nanostructures can provide different thermoelectric properties of the nanoribbons. They can also provide more realistic simulations for the actual experimental tests ${ }^{73}$. Nonetheless, the calculation of the spin thermoelectric properties of the considered GSNRs using experimental methods is beyond the scope of the present research. Although there may be a difference between the results obtained by this research with those given by the actual experimental tests, the results of this study can suitably pave the way for the development of standard experimental methods and thermoelectric property assessment of the GSNRs. It is concluded that a nearly full spin filtering effect with spin-dependent localized transmission peaks near the chemical potential $\mu=0$ can be obtained. Moreover, larger spin-dependent Seebeck coefficient and spin polarization efficiency are identified compared to those given by ${ }^{74-76}$. To further study the thermoelectric properties of the GSNRs, three defect arrangements with different orientations are also considered for the larger case study, and their responses are re-evaluated. The results of the present study are summarized in the subsequent sections.

\section{Device structure and computational details}

Three different parallel step-like GSNR junctions are introduced using semi-infinite metallic AGNRs with different widths of the left and right leads, and a ZSNR in the central region ${ }^{43,77}$. Each left and right lead is generated by duplicating two different unit cells along the transport direction. As shown in Fig. 1, each system contains $2 N_{\mathrm{AG}}=46,70$, and 94 carbon atoms in each unit cell in the left and $N_{\mathrm{AG}}-1$ in the right region. $N_{\mathrm{AS}}=6,10$, and 14 number of armchair silicon-atom chains are also considered for the central regions. The changes in the central region width are dependent on the left contact size. These step-like GSNR junctions are named 23GE-6SE-11GE, $35 \mathrm{GE}-10 \mathrm{SE}-17 \mathrm{GE}$, and 47GE-14SE-23GE, respectively. To further study the divacancy effects on the electronic and thermoelectric properties of the selected devices, three different defect orientations are considered in the central scattering region. In this study, the pentagon-octagon-pentagon (5-8-5) defect type ${ }^{78,79}$ is selected for the defective system. The DVs are also assumed to be duplicated with the periodicity of $l=4^{80}$. Despite a DV in the nanostructure, an equal number of A- and B-sublattice sites are assumed in all cases (see Fig. 1). This can provide net magnetic moment per unit cell i.e., $m=\left(N_{\mathrm{A}}-N_{\mathrm{B}}\right) \mu_{B}=0^{81}$.

To obtain the spin caloritronic characteristics of the selected nanostructures, their electron transmissions are first determined using the NEGF method ${ }^{82}$ and Landauer-Bütticker transport formula ${ }^{83}$. Because there is a weak coupling among the electrons and phonons, the electron-phonon interactions are ignored in this study ${ }^{84-86}$. Thus, electronic transports can appropriately be assumed ballistic. The spin-flip scattering is also neglected. Hence, the spin-up and spin-down electron transports can singly be investigated. This is valid because the spin diffusion length in SE is in the order of several micrometers ${ }^{87}$. As shown in Fig. 1, the system is divided into three various segments, including the left and right electrodes, and the central region. The generalized Hamiltonian for the system consists of several submatrices as follows:

$$
H_{\mathrm{T}}=H_{\mathrm{L}}+H_{\mathrm{R}}+H_{\mathrm{C}}+H_{\mathrm{CL}}+H_{\mathrm{CR}} \text {, }
$$

where $H_{\mathrm{L}}, H_{\mathrm{R}}$, and $H_{\mathrm{C}}$ are the Hamiltonian matrices of the isolated left electrode, right electrode, and the central region, respectively. These submatrices can be determined by using Eqs. (2) and (3). In Eq. (4), $H_{\mathrm{CL}}$ and $H_{\mathrm{CR}}$ 

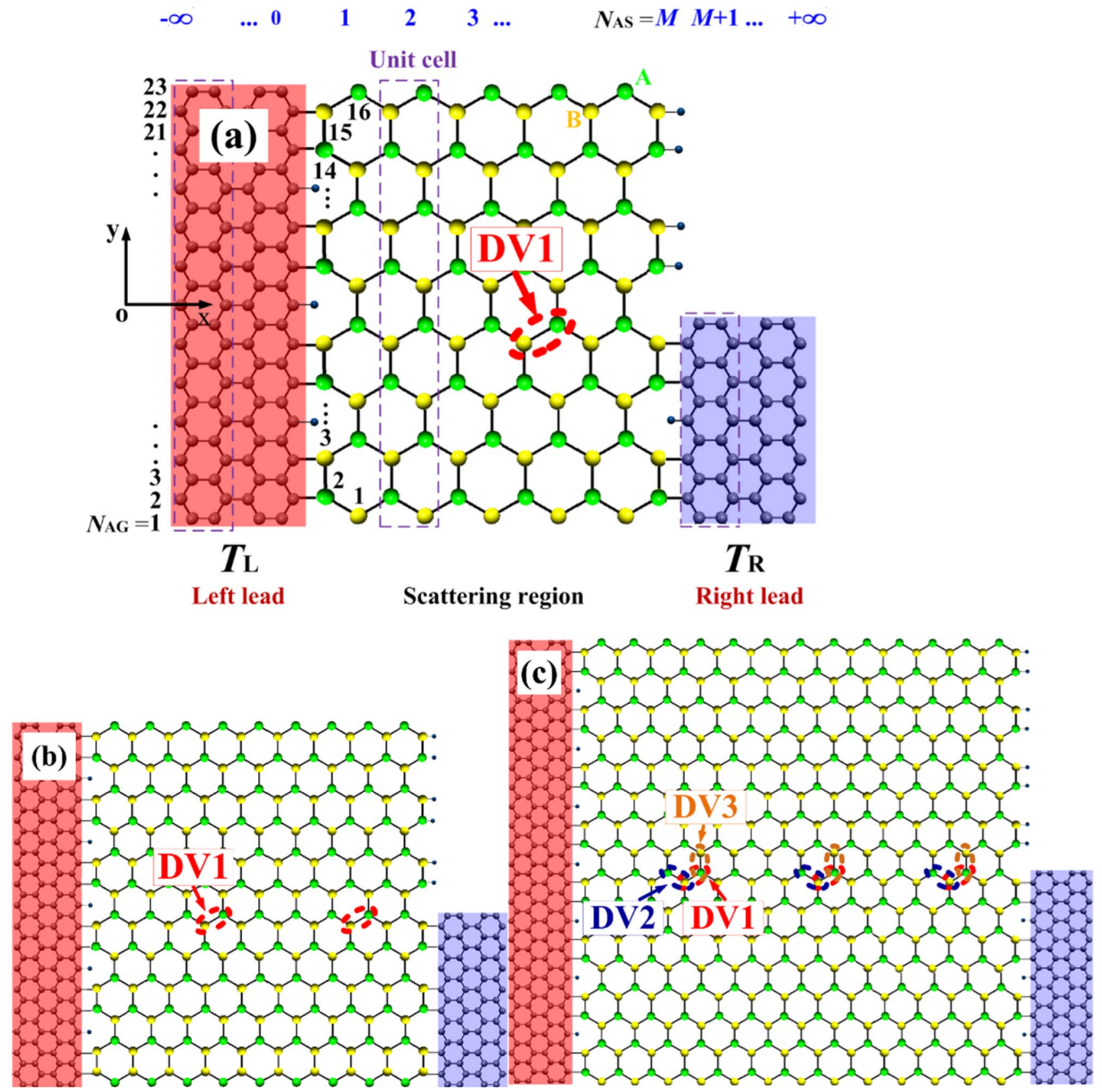

Figure 1. Schematic view of the defected parallel step-like GSNR junctions for (a) the 23GE-6SE-11GE, (b) the $35 \mathrm{GE}-10 \mathrm{SE}-17 \mathrm{GE}$, and (c) the $47 \mathrm{GE}-14 \mathrm{SE}-23 \mathrm{GE}$ case studies. Each unit cell of the left lead has $2 N_{\mathrm{AG}}$ atoms. The central region is composed of about $N_{\mathrm{AS}}$ unit cells. Different DV orientations are also represented in the 47GE-14SE-23GE.

are also defined by the hopping between the central region and the left and right electrodes, respectively. The Hamiltonian matrices are all determined using the TB method ${ }^{88-90}$.

$$
\begin{gathered}
H_{\mathrm{R}(\mathrm{L})}=-t_{\mathrm{R}(\mathrm{L})} \sum_{\langle i, j\rangle, \alpha} c_{i \alpha}^{\dagger} c_{j \alpha}+\mathrm{e} E_{Y \mathrm{G}} \sum_{i, \alpha} Y_{i \mathrm{G}} c_{i \alpha}^{\dagger} c_{i \alpha}+\text { H.c., } \\
H_{\mathrm{C}}=-t_{\mathrm{C}} \sum_{\langle i, j\rangle, \alpha} c_{i \alpha}^{\dagger} c_{j \alpha}+i \frac{\lambda_{s o}}{3 \sqrt{3}} \sum_{\ll i, j \gg, \alpha, \beta} v_{i j} c_{i \alpha}^{\dagger}\left(\sigma_{z}\right)_{\alpha \beta} c_{j \beta}+M_{Z} \sum_{i, \alpha} c_{i \alpha}^{\dagger} \sigma_{z} c_{i \alpha} \\
+\mathrm{e} l E_{Z} \sum_{i, \alpha} \xi_{i} c_{i \alpha}^{\dagger} c_{i \alpha}+\mathrm{e} E_{Y S} \sum_{i, \alpha} Y_{i S} c_{i \alpha}^{\dagger} c_{i \alpha}+\text { H.c., }
\end{gathered}
$$




$$
H_{\mathrm{CR}(\mathrm{CL})}=-t_{\mathrm{CR}(\mathrm{CL})} \sum_{\langle i, j\rangle, \alpha} c_{i \alpha}^{\dagger} c_{j \alpha}+\text { H.c., }
$$

in which $t_{\mathrm{R}(\mathrm{L})}$ and $t_{\mathrm{C}}$ are the hopping energies for the nearest-neighbor interactions in the right (left) lead, and the central region, respectively. In this study, 2.66 and $1.60 \mathrm{eV}$ values are assumed for $t_{\mathrm{R}(\mathrm{L})}$ and $t_{\mathrm{C}}$, respectively ${ }^{88,90}$. $c_{i \alpha}^{\dagger}\left(c_{i \alpha}\right)$ denotes the fermion creation (annihilation) operator at site $i . \alpha$ also is the spin index. $<i, j>$ and $<<i$, $j>>$ show the nearest-neighbor and second-nearest-neighbor atoms, respectively. The rest of parameters used in Eqs. (2) - (4) are defined as follows: $\lambda_{s o}$ is the effective spin-orbit interaction and is assumed equal to $3.9 \mathrm{meV}$ and zero for the SE and GE nanoribbons, respectively ${ }^{89} . \sigma$ is the Pauli matrix. $v_{i j}$ is a parameter equal to $-1(+1)$ for the clockwise (counterclockwise) hopping index about the $\mathrm{Z}$-axis. $\xi_{i}=+1,-1$ is the valley index for the sublattices $A$ and $B$, respectively. $E_{Z}$ is a perpendicular electric field that can yield a voltage difference equal to $2 \mathrm{e} l E_{Z}$ in which e is the electron charge and $l$ is the half distance of the two sublattices. $M_{Z}$ is the exchange field induced by the proximity effect of ferromagnetic material ${ }^{91} . E_{Y G}$ and $E_{Y S}$ are the inhomogeneous transverse electric fields along $y$-direction, and applied to the leads and central region, respectively. $Y_{i \mathrm{G}}$ and $Y_{i \mathrm{~S}}$ denote the $y$-coordinate of the atom $i$ in the SE and GE nanoribbons. In this study, the electric and ferromagnetic exchange fields are assumed to be $0.082 \mathrm{~V} / \AA$ and $0.162 \mathrm{eV}$, respectively, and perpendicularly applied to the central region of the hybrid GSNRs. The effects of inhomogeneous transverse electric fields $E_{Y S}=0.917 \mathrm{~V} / \AA$ and $E_{Y G}=0.680 \mathrm{~V} / \AA$ are also considered. $t_{\mathrm{CR}}\left(t_{\mathrm{CL}}\right)$ denotes the hopping energy between the central region and right (left) lead, respectively. In this study, Harrison's scaling law ${ }^{92,93}$ is used to compute the contact hopping energies for the GE and SE at the interface. At the interface, the bond length between carbon and silicon atoms is $1.89 \AA$, and its corresponding hopping energy is calculated as $t_{\mathrm{CR}(\mathrm{CL})}=1.88 \mathrm{eV}$. It is worth mentioning that this value needs to be in the same order of $t_{\mathrm{R}(\mathrm{L})}$ and $t_{\mathrm{C}}{ }^{4}$. The spin-dependent transmission function, $T_{\alpha}$, is used to determine the thermoelectric properties of the nanostructures, and can be obtained by Eq. (5) and using NEGF formalism ${ }^{94}$ :

$$
T_{\alpha}(E)=\operatorname{Tr}\left[\Gamma_{\mathrm{L}, \alpha}(E) \mathrm{G}_{\mathrm{C}, \alpha}(E) \Gamma_{\mathrm{R}, \alpha}(E) G_{\mathrm{C}, \alpha}^{\dagger}(E)\right],
$$

where $\Gamma_{R(L), \alpha}=i\left(\sum_{R(L), \alpha}-\sum_{R(L), \alpha}^{\dagger}\right)$ is the interaction between the right (left) lead with the scattering region, in which $\Sigma_{\mathrm{R}(\mathrm{L}), \alpha}$ is the right (left) self-energy ${ }^{94}$. $\Sigma_{\mathrm{R}(\mathrm{L}), \alpha}$ includes the effect of two semi-infinite AGNRs on the scattering region. $G_{C, \alpha}(E)$ also denotes the retarded spin Green's function of the scattering region, and can be expressed as follows ${ }^{94}$ :

$$
G_{\mathrm{C}, \alpha}=\left[\left(E+\mathrm{i}^{+}\right) \mathrm{I}-H_{\mathrm{C}}-\sum_{\mathrm{L}, \alpha}-\sum_{\mathrm{R}, \alpha}\right]^{-1},
$$

The temperatures in the left and right leads are set to $T_{\mathrm{L}}$ and $T_{\mathrm{R}}$, respectively. Hence, the temperature difference can be obtained by $\Delta T=T_{\mathrm{L}}-T_{\mathrm{R}}$. Based on the Landauer-Büttiker formula, the spin-dependent current can be obtained by ${ }^{95}$ :

$$
I_{\alpha}=\frac{\mathrm{e}}{\mathrm{h}} \int_{-\infty}^{+\infty} T_{\alpha}(E)\left[f_{\mathrm{L}}\left(E, T_{\mathrm{L}}\right)-f_{\mathrm{R}}\left(E, T_{\mathrm{R}}\right)\right] d E
$$

where e and $\mathrm{h}$ are the electron charge and the Plank constant, respectively. $f_{\mathrm{R}(\mathrm{L})}$ and $T_{\mathrm{R}(\mathrm{L})}$ represent the average Fermi-Dirac distribution and the temperature for the right (left) lead, respectively. $T_{\alpha}$ is also the spin-dependent transmission. The net spin current is defined as $I_{s}=I_{u p}-I_{d n}$ and calculated by Eq. (7). Considering a linear response regime (i.e., $\Delta T \cong 0$ ), the spin-dependent Seebeck coefficient can then be computed for each spin channel as follows ${ }^{96}$ :

$$
S_{\alpha}=-\frac{1}{\mathrm{e} T}\left(\frac{L_{1, \alpha}}{L_{0, \alpha}}\right) .
$$

where $L_{n, \alpha}$ is the intermediate function and defined as ${ }^{95}$

$$
L_{n, \alpha}(\mu, T)=-\frac{1}{h} \int(E-\mu)^{n} \frac{\partial f(E, \mu, T)}{\partial E} T_{\alpha}(E) d E,
$$

where $\mu$ is the chemical potential. The spin and charge Seebeck coefficients can then be computed by $S_{s}=S_{u p}-S_{d n}$ and $S_{c}=\left(S_{u p}+S_{d n}\right) / 2$, respectively ${ }^{96}$. The electrical conductance is also defined as

$$
\mathrm{G}_{\alpha}=-e^{2}\left(L_{0, \alpha}\right) \text {. }
$$

where $G_{c}=G_{u p}+G_{d n}$ and $G_{s}=\left|G_{u p}-G_{d n}\right|$ is the charge and spin thermal conductance, respectively ${ }^{97}$. The spindependent electron thermal conductance is obtained as ${ }^{98}$

$$
\begin{gathered}
\kappa_{\mathrm{e}, \alpha}=\frac{1}{T}\left(L_{2, \alpha}-\frac{L_{1, \alpha}^{2}}{L_{0, \alpha}}\right) . \\
Z_{\mathrm{e}, c(s)} T=S_{c(s)}^{2} \mathrm{G}_{c(s)} T / \kappa_{\mathrm{e}} .
\end{gathered}
$$



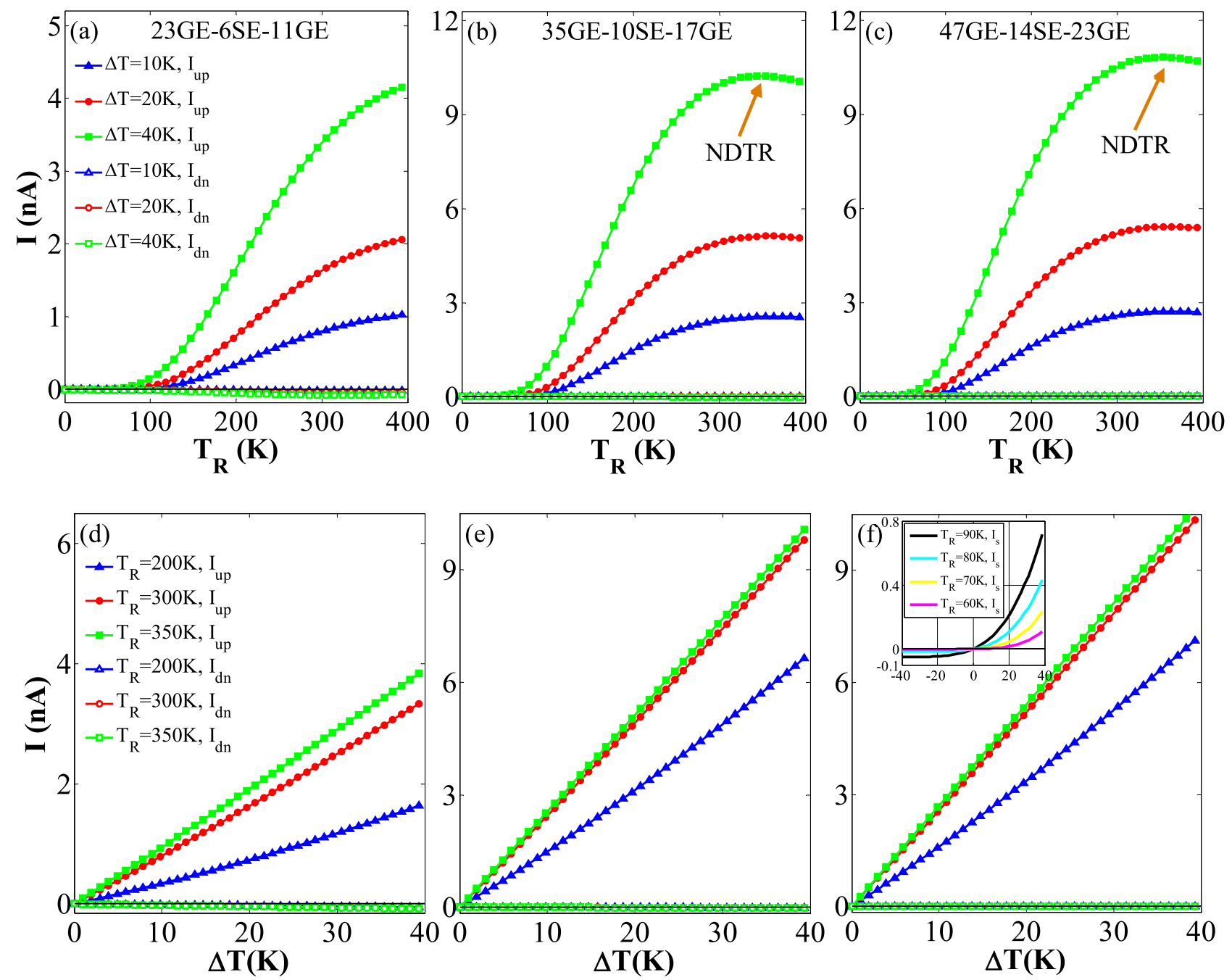

Figure 2. Panels (a), (b), and (c) show the variation of the spin-polarized currents $\left(I_{u p}\right.$ and $\left.I_{d n}\right)$ against $T_{\mathrm{R}}$ for $\Delta T=10,20$, and $40 \mathrm{~K}$; Panels (d), (e), and (f) show $I_{u p}$ and $I_{d n}$ against $\Delta T$ for $T_{\mathrm{R}}=200,250$, and $350 \mathrm{~K}$ for the 23GE-6SE-11GE, 35GE-10SE-17GE, and 47GE-14SE-23GE configurations, respectively.

where $Z_{\mathrm{e}} T$ is the electrical FOM and is defined as the upper limit of $Z T=S^{2} \mathrm{GT} /\left(\kappa_{\mathrm{e}}+\kappa_{\mathrm{ph}}\right)$ value, in which $\kappa_{\mathrm{ph}}$ and $\kappa_{\mathrm{e}}$ are the phonon's and electron thermal conductance, respectively. The maximum value of $Z T$ occurs when $\kappa_{\mathrm{ph}}$ is very smaller than $\kappa_{\mathrm{e}}^{99,100}$.

Analyses and results. To numerically investigate the performance of the step-like GSNRs, three different length-to-width ratios $(\beta)$ of the central region, i.e., $\beta \cong 0.9,1.0$, and 1.1 , are selected for the considered devices (see Fig. 1). To produce a step-like nanoribbon, it is assumed that the right contact in all cases has a half-width from the left one. Each device is subjected to perpendicular electric $\left(E_{\mathrm{Z}}\right)$ and ferromagnetic exchange $\left(M_{\mathrm{Z}}\right)$ fields in the central region. It is noted that both local $E_{Z}$ and $M_{Z}$ fields ${ }^{101}$ can be produced in the laboratory environment. The effect of inhomogeneous transverse electric fields, i.e., $E_{Y S}$ and $E_{Y G}$ are also included for each system, respectively ${ }^{102}$. The electric current is also calculated by applying a difference between the temperature of the left, $T_{\mathrm{L}}$, and the right, $T_{\mathrm{R}}$, electrodes with no back gate voltage, and the spin currents are only determined in the presence of the temperature gradient.

In each system, a temperature gradient is generated between the two electrodes using $\Delta T>0$. This can cause a nonzero value of $f_{\mathrm{L}}-f_{\mathrm{R}}$, and produce a spin-dependent current depending on $T_{\mathrm{R}(\mathrm{L})}$ and $\Delta T$. In addition, it creates changes in the spin-up and spin-down currents in the GSNRs at different step-like junctions. The thermal SFE is studied for the considered hybrid step-like GSNRs with threshold temperature $\left(T_{\mathrm{th}}\right)$. The variations of the spin-dependent current $I_{u p}\left(I_{d n}\right)$ versus $T_{\mathrm{R}}$ with $\Delta T=10,20$, and $40 \mathrm{~K}$ are shown in Fig. $2 \mathrm{a}-\mathrm{c}$, representing small to large step-like GSNRs. As shown in these figures, when $T_{R}$ increases, $I_{u p}$ reaches its maximum value for the high temperatures, whereas $I_{d n}$ value is almost zero and remains unchanged for the whole temperature range. Moreover, the spin-up current is positive $\left(I_{u p}>0\right)$ for almost $T_{R}>100 \mathrm{~K}$, while the spin-down current $I_{d n} \cong 0$ throughout. The results show that the considered GSNRs can well show an insulating response with no charge or spin current for nearly $T_{R}<100 \mathrm{~K}$. For example, the threshold temperature value $T_{\text {th }}=50 \mathrm{~K}$ occurs at $\Delta T=40 \mathrm{~K}$ 

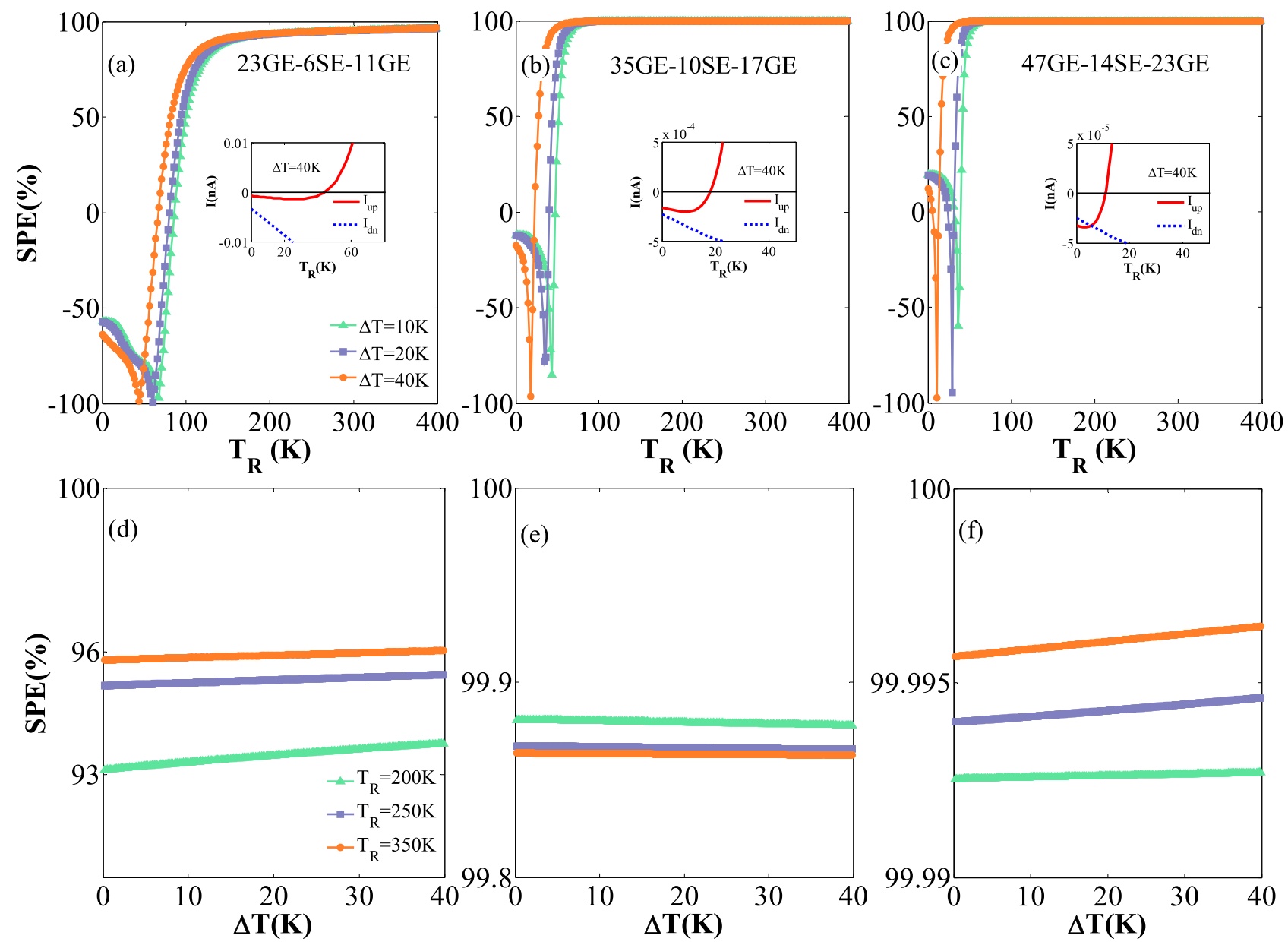

Figure 3. Panels (a), (b), and (c) show the variation of the SPE against $T_{\mathrm{R}}$ for $\Delta T=10,20$, and $40 \mathrm{~K}$; Panels (d), (e), and (f) show the SPE variation against $\Delta T$ for $T_{\mathrm{R}}=200,250$, and $350 \mathrm{~K}$ for the 23GE-6SE-11GE, 35GE-10SE17GE, and 47GE-14SE-23GE configurations, respectively. The insets show the $I_{u p}$ reverse sign and the mutual competition among $I_{u p}$ and $I_{d n}$ for $T_{\mathrm{R}}<100 \mathrm{~K}$ and $\Delta T=40 \mathrm{~K}$.

for the 23GE-6SE-11GE, whereas this value for the 35GE-10SE-17GE and the 47GE-14SE-23GE cases is similarly equal to $30 \mathrm{~K}$. In addition, $T_{\mathrm{th}}$ marginally decreases by increasing the GSNR size. This is dependent on where the peak of spin-up transmission occurs as the system size changes (see Fig. 4). Thus, $T_{\text {th }}$ value can be controlled by changing the system size. This clearly illustrates that the SFE ${ }^{15}$ is accompanied by the spin switching effect in these cases. As shown in Fig. 2b,c, the SFE is stronger when the device size increases. The peak values of $I_{u p}$ are almost obtained as 4.2,10.24, and $10.83 \mathrm{nA}$ for the 23GE-6SE-11GE, 35GE-10SE-17GE, and 47GE-14SE-23GE case studies, respectively (see Fig. 2a-f). Thus, the strength of the current varies when the device size is changed. However, the NDTR happens for the higher temperatures and $\Delta T$. Accordingly, the hybrid step-like GSNRs can be utilized as a thermal spin device with multiple different characteristics. Figure $2 \mathrm{~d}-\mathrm{f}$ also show the changes of $I_{u p}$ and $I_{d n}$ against $\Delta T$ for $T_{\mathrm{R}}=200,250$, and $350 \mathrm{~K}$, respectively. These figures demonstrate that $I_{u p}$ increases as $T_{\mathrm{R}}$ and $\Delta T$ increase, while $I_{d n}$ keeps nearly zero for various $\Delta T$ values. This again confirms that the thermal SFE has been generated. A thermal rectification behavior is also observed for the lower $T_{\mathrm{R}}$ and larger size of devices (see inset of Fig. 2f).

Figure 3 shows the variation of the spin polarization efficiency SPE $\left(=\left(\left|I_{u p}\right|-\left|I_{d n}\right|\right) /\left(\left|I_{u p}\right|+\left|I_{d n}\right|\right) \times 100 \%\right)$ versus $T_{\mathrm{R}}$ and $\Delta T$ for the studied systems. The results illustrate that the considered step-like GSNR junctions can suitably provide a thermal spin current with high SPE. The SPE of the devices is often dependent on the temperature sets and, most notably, on their sizes. Therefore, it is not appropriate to study the SPE below the 'on-off' temperature for the spin-up currents, in which the spin-up and spin-down channels are closed. The results show that a nearly $100 \%$ SPE can be achieved for a broad range of $T_{\mathrm{R}}$ and $\Delta T$ values. This is especially true for the 47GE-14SE$23 \mathrm{GE}$ configuration (see Fig. 3e,f). Figure 3 also shows that SPE experiences sudden changes for a limited range of temperatures in some cases. This is due to the $I_{u p}$ reverse sign and the mutual competition among $I_{u p}$ and $I_{d n}$ within this range of temperatures (See insets in Fig. 3). The SPE values larger than $99.80 \%$ and $99.99 \%$ can also be observed for the second and third case studies for the different values of $\Delta T$ at $T_{R}=200 \mathrm{~K}$, respectively, whereas a nearly $93.0 \%$ value is obtained for the first case. This evidence confirms that the GNR and SNR-based devices often show less SPE values than their corresponding studied counterparts ${ }^{74,75,103}$. The results of numerical analyses clearly show that the response of a hybrid nanostructure strongly depends on the values selected for the external 


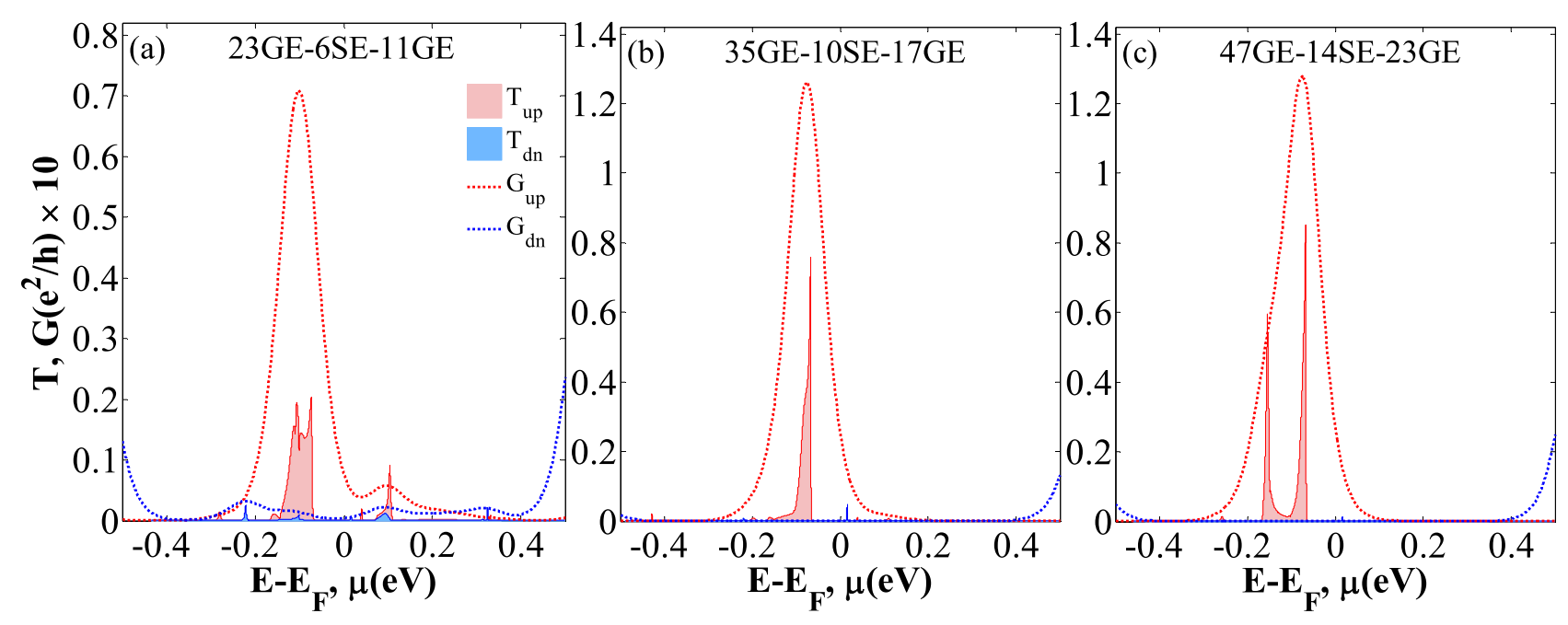

Figure 4. Panels (a), (b), and (c) show the variation of the spin-dependent transmission spectra $\left(T_{u p}\right.$ and $\left.T_{d n}\right)$ against $E-E_{\mathrm{F}}$ (filled area) and dotted lines show the variation of the spin-dependent electrical conductance $\left(G_{u p}\right.$ and $\left.G_{d n}\right)$ versus $\mu$ for the 23GE-6SE-11GE, 35GE-10SE-17GE, and 47GE-14SE-23GE configurations, respectively, at $T=300 \mathrm{~K}$.

electric and ferromagnetic exchange fields. The value of the fields in the present study has been chosen in such a way that we obtain the SFE and maximize its efficiency. The results demonstrate that the selected magnitudes can provide high-efficiency value up to $99.9 \%$ in some case studies. It is noted that such a high SFE value has previously been obtained and reported by other researchers (e.g., $\left.{ }^{104-107}\right)$.

The changes of the spin-polarized transmission coefficients $\left(T_{u p}\right.$ and $\left.T_{d n}\right)$ against energy $\left(E-E_{F}\right)$ and spindependent electrical conductance $\left(G_{u p}\right.$ and $\left.G_{d n}\right)$ versus the chemical potential $(\mu)$ for the three studied cases are displayed in Fig. 4. In these plots, $T_{u p}\left(T_{d n}\right)$ and $G_{u p}\left(G_{d n}\right)$ are shown by a filled area and dotted line, respectively, and the Fermi level is set to zero. As shown in Fig. 4, the transmission peak values almost locally occur around the Fermi level i.e., the peak value of $T_{u p}$ almost occurs at $[-0.16 \mathrm{eV},-0.06 \mathrm{eV}],[-0.001 \mathrm{eV},-0.07 \mathrm{eV}]$, and $[-0.17 \mathrm{eV},-0.07 \mathrm{eV}]$ intervals, for the 23GE-6SE-11GE, 35GE-10SE-17GE, and 47GE-14SE-23GE configurations, respectively. Nonetheless, they do not include a strong peak value for $T_{d n}$ around the Fermi level. This is particularly true for the second and third configurations. This confirms that the spin-up governs the transport properties with $I_{u p}>0$, and can generate an almost perfect thermal SFE. The spin gap along with the narrow transmission peaks near the chemical potential $\mu=0 \mathrm{eV}$ can lead to a larger spin-dependent Seebeck coefficient compared to the values provided by the GNRs and $\mathrm{SNRs}^{108,109}$. Figure 4 clearly shows that $G_{d n}$ is nearly zero in the spin-down channel for some ranges of $\mu$ values, whereas $G_{u p}$ is conductive in the spin-up channel. As a result, the proposed nanostructure device can provide a fully spin-polarized current.

Figure 5 shows the variation of $S_{u p}, S_{d n}, S_{s}$, and $S_{c}$ versus the chemical potential $(\mu)$ at $T_{R}=300 \mathrm{~K}$. As shown in Fig. 5, the response of $S_{u p}\left(S_{d n}\right)$ is not very identical around $\mu=0 \mathrm{eV}$ for various sizes of nanoribbons. For each configuration, there are also some points at a given chemical potential value in which $S_{\alpha}$ (or thermal spin current) is equal to zero for one spin channel and is not zero for the other one; because the electron and hole currents are neutralized by one another. Hence, a perfect spin-polarized current can be obtained by the thermal gradient. Based on the results, the peak values of $S_{s}$ around $\mu=0 \mathrm{eV}$ are obtained as $0.404,0.491$, and $0.547 \mathrm{mV} / \mathrm{K}$ for the $23 \mathrm{GE}-6 \mathrm{SE}-11 \mathrm{GE}, 35 \mathrm{GE}-10 \mathrm{SE}-17 \mathrm{GE}$, and $47 \mathrm{GE}-14 \mathrm{SE}-23 \mathrm{GE}$ case studies, respectively ${ }^{76}$. The results also show that $S_{s}$ can be increased as the device size is increased. This may be attributed to the fact that the spin gap gets larger. The peak value of $\left|S_{s}\right|$ occurs in the large chemical potential region and almost around $\mu= \pm 0.4 \mathrm{eV}$, while the location of these peak values almost remained unchanged when the device size increases. The maximum values of $\left|S_{s}\right|$ for the 23GE-6SE-11GE, 35GE-10SE-17GE, and 47GE-14SE-23GE cases are obtained as 0.826, 1.150, and $1.114 \mathrm{mV} / \mathrm{K}$, respectively. These values are very larger than the values reported in other researches (e.g., $S_{s}$ $\sim 300 \mu \mathrm{V} / \mathrm{K}$ in ${ }^{97,98}, S_{s} \sim 550 \mu \mathrm{V} / \mathrm{K} \mathrm{in}^{76}$, and $S_{s} \sim-90 \mu \mathrm{V} / \mathrm{K}$ in $\left.^{108}\right)$. The color of $S_{s}$ varies in accordance with the Seebeck polarization, $P_{s}\left(=\left(\left|S_{u p}\right|-\left|S_{d n}\right|\right) /\left(\left|S_{u p}\right|+\left|S_{d n}\right|\right)\right)$ in Fig. 5. As shown in Fig. 5, $S_{s}$ color varies from orange to black, representing the contribution of the spin-down and spin-up electrons in $S_{s}$, respectively. The charge and spin thermoelectric efficiencies are also assessed by calculating the electrical FOM $\left(Z_{\mathrm{e}, c} T\right.$ and $\left.Z_{\mathrm{e}, s} T\right)$ for each case study and different $\mu$ values at room temperature (See insets of Fig. 5). The results show that the thermoelectric efficiency of a given device can significantly be improved as the size changes. The maximum values of the charge (spin) thermoelectric efficiencies occur about 15.06 (101.4), 13.4 (49.2), and 4.5 (9.2) from the large to small devices, respectively. It is realized that the third nanostructure case study (i.e., 47GE-14SE-23GE) can provide the largest charge and spin thermoelectric efficiency and enhance $Z_{e} T$. This enhancement is very larger than that reported in ${ }^{110}$. The obtained results illustrate that the engineering defects in step-like GSNRs can provide a quite acceptable spin thermoelectric efficiency. It is noted that $Z_{\mathrm{e}} T$ is always larger than $Z T$. Hence, high value for $Z_{\mathrm{e}} T$ is nonsense in reality. Low $Z_{\mathrm{e}} T$ values are important because they allow us to exclude unpromising materials. 


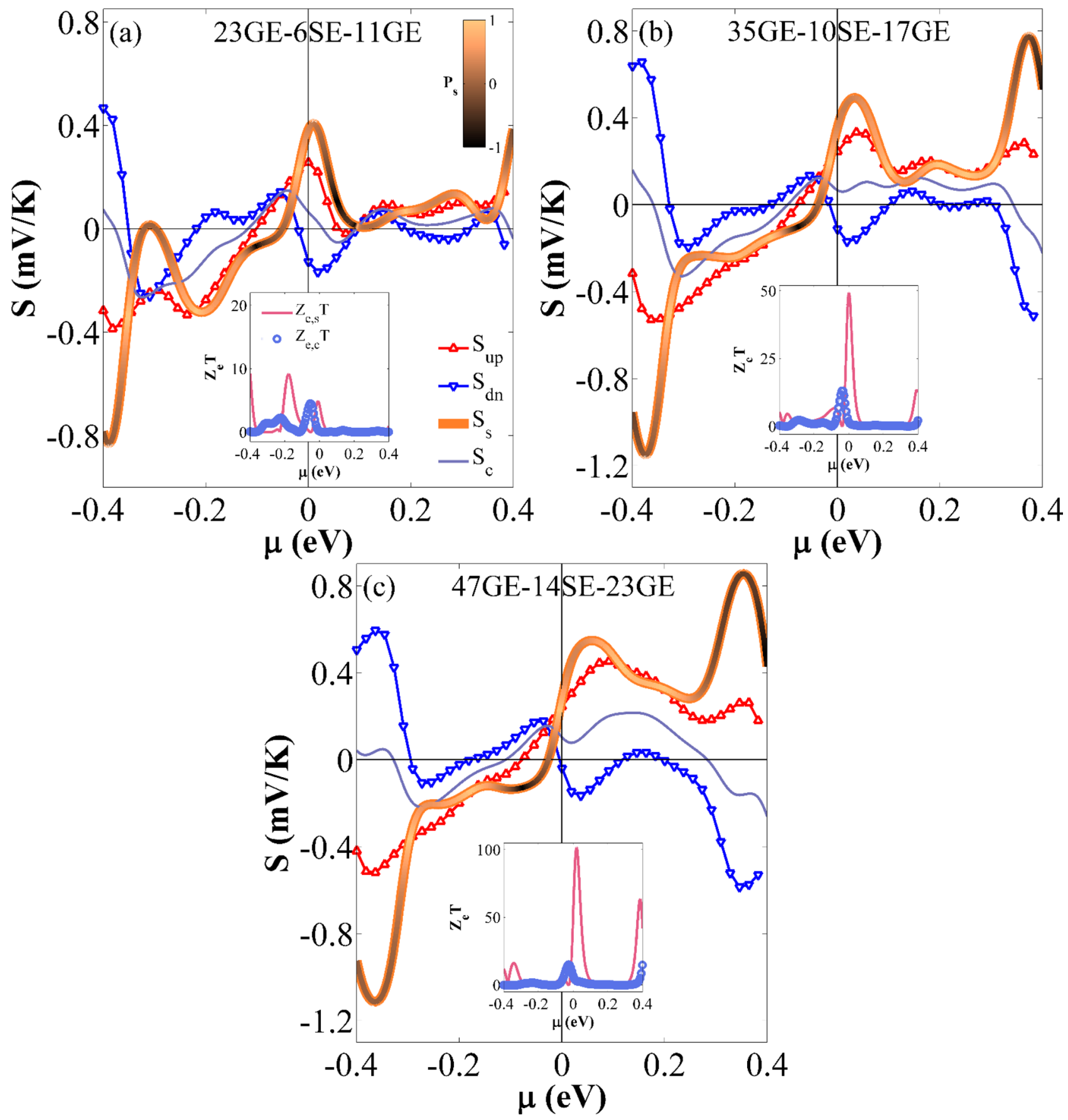

Figure 5. The $S_{u p}, S_{d n}, S_{s}$, and $S_{c}$ against $\mu$ at room temperature for (a), (b), and (c) 23GE-6SE-11GE, $35 \mathrm{GE}-10 \mathrm{SE}-17 \mathrm{GE}$, and $47 \mathrm{GE}-14 \mathrm{SE}-23 \mathrm{GE}$ configurations, respectively. $S_{S}$ color varies based on Seebeck polarization $\left(P_{s}\right)$ value. The insets show the $Z_{\mathrm{e}, s} T$ and $Z_{\mathrm{e}, c} T$ as a function of $\mu$.

The effect of DV orientation on spin caloritronics. In order to achieve complete spin-dependent thermoelectric properties of the step-like GSNR junctions, a set of DVs with three different orientations in the 47GE-14SE-23GE (i.e., DV1, DV2, and DV3) is created and are analytically assessed ${ }^{78,111}$ (see Fig. 1c). The variation of $I_{u p}$ and $I_{d n}$ against $T_{\mathrm{R}}$ for DV1, DV2, and DV3 cases and $\Delta T=40 \mathrm{~K}$ are displayed in Fig. $6 . I_{u p}$ and $I_{d n}$ values for no vacancy (NV) defect configuration are also plotted for the sake of comparison. As shown in the inset of Fig. 6a, the spin and charge transporting channels are almost closed for the NV and DV3 cases for the entire temperature ranges. In contrast, the other cases (i.e., DV1 and DV2) respond as an insulator for $T_{\mathrm{R}}<40 \mathrm{~K}$. In DV1 and DV2 cases, $I_{d n}$ almost remained unchanged and equal to zero for the entire range of temperatures, while $I_{u p}$ is significantly increased as $T_{\mathrm{R}}$ exceeds $40 \mathrm{~K}$. This confirms that the SFE is along with the spin switching effect in these cases, and occur only due to the temperature gradient with no back gate voltage. The NDTR phenomenon occurs for the latter two cases. For each case, $I_{u p}$ absolute value also increases by $T_{R}$, while their flowing directions 

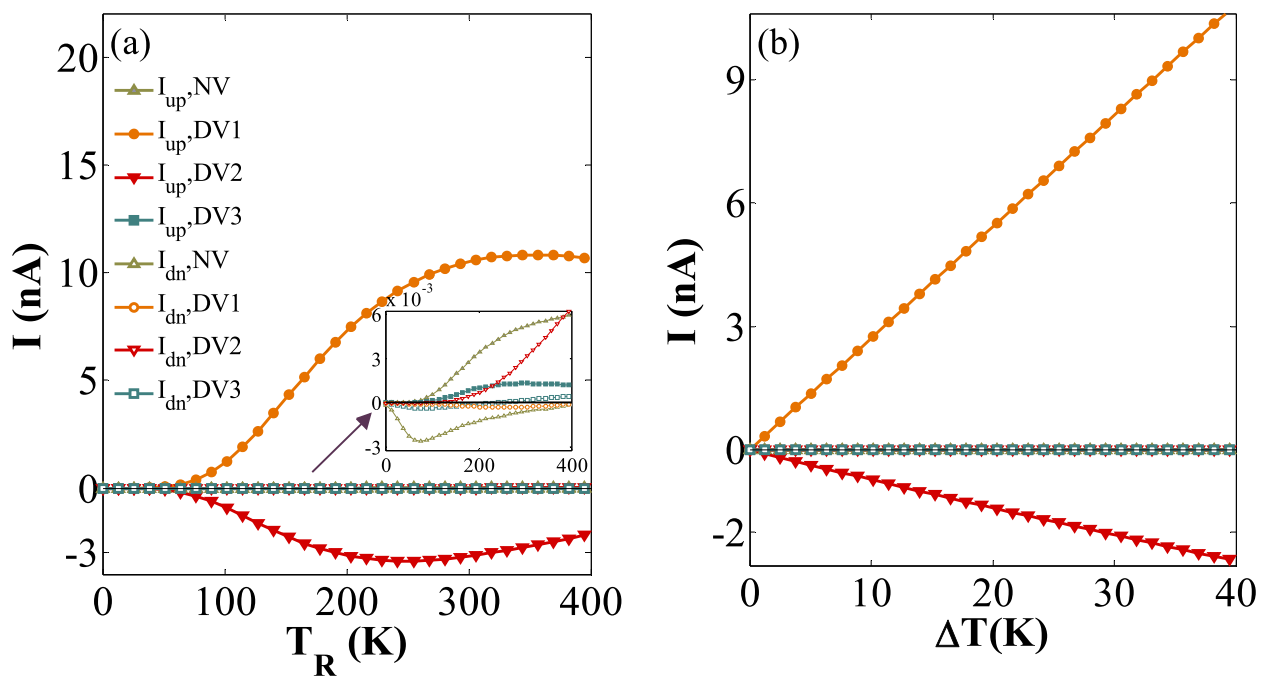

Figure 6. (a) $I_{u p}$ and $I_{d n}$ versus $T_{\mathrm{R}}$ for $\Delta T=40 \mathrm{~K}$; (b) $I_{u p}$ and $I_{d n}$ versus $\Delta T$ for $T_{\mathrm{R}}=350 \mathrm{~K}$ and NV, DV1, DV2, and DV3 configurations, respectively.

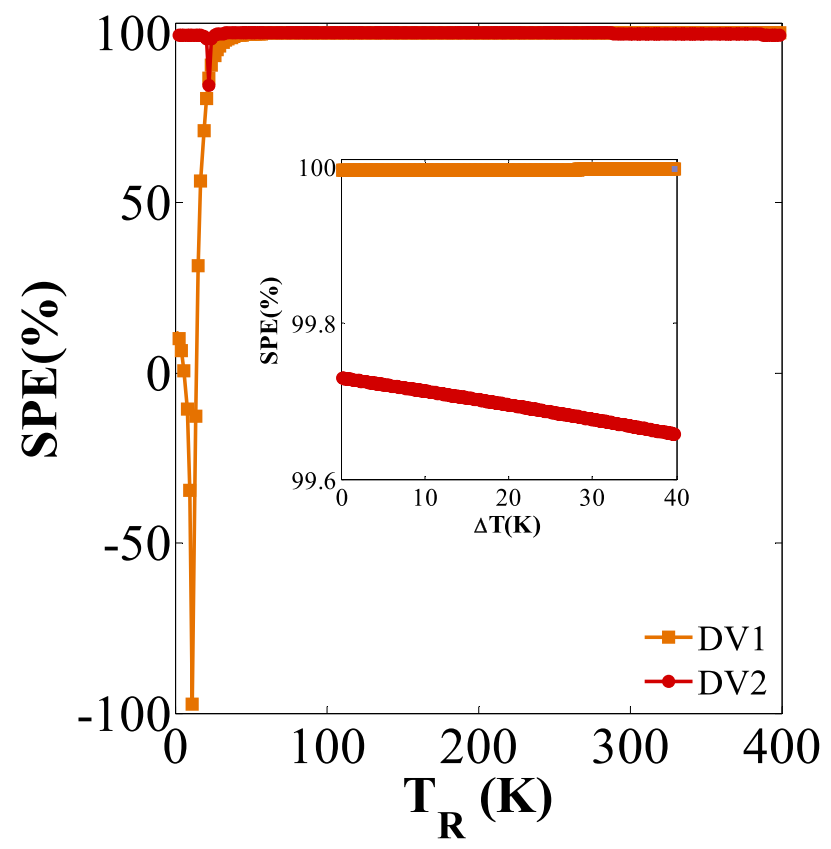

Figure 7. The SPE versus $T_{R}$ for $\Delta T=40 \mathrm{~K}$; The inset shows SPE versus $\Delta T$ for $T_{R}=350 \mathrm{~K}$; for DV1and DV2 configurations, respectively.

are opposite at a given $T_{\text {th }}$. Figure $6 \mathrm{~b}$ also presents the variation of $I_{u p}\left(I_{d n}\right)$ against $\Delta T$ for $T_{R}=350 \mathrm{~K}$. As shown in Fig. $6 \mathrm{~b}, I_{u p}$ almost linearly increases for the DV1, whereas it is decreased for the DV2.

The variations of SPE against $T_{\mathrm{R}}$ and $\Delta T$ are also separately plotted for DV1 and DV2 case studies in Fig. 7. $\Delta T=40 \mathrm{~K}$ and $T_{\mathrm{R}}=350 \mathrm{~K}$ are assumed for these plots, respectively. An SPE value larger than $99.6 \%$ can be obtained for DV2 case and a broad range of $T_{\mathrm{R}}$ and $\Delta T$ values. This shows a nearly $0.5 \%$ SPE value difference between DV1 and DV2 ${ }^{78}$. In the DV2 case, the maximum SPE value occurs for high $T_{\mathrm{R}}$ and low $\Delta T$ values. This is fully compatible with the results reported in Fig. 6. It is worth mentioning that a sudden drop can be seen in Fig. 7 for a limited range of $T_{\mathrm{R}}$ values. However, this is negligible and is not essential in practical applications.

Figure 8 shows the variations of $T_{u p}\left(T_{d n}\right)$ against $E-E_{F}$ and $G_{u p}\left(G_{d n}\right)$ versus $\mu$ for DV1 and DV2 cases. The plot indicates that the variation of DV orientation can change the values of $T_{u p}\left(T_{d n}\right)$ and $G_{u p}\left(G_{d n}\right)$, while keeping the spin-up electron as the dominant carrier in both cases. Comparing DV1 and DV2 cases shows that $T_{u p}$ is remarkably enhanced when the divancancy orientation is changed to DV1. Although the peak values occur around $\mu=0 \mathrm{eV}$ in both cases, there are two peaks for DV1 case at $[-0.17 \mathrm{eV},-0.07 \mathrm{eV}]$, whereas two peak values for DV2 case are in both sides of this point, i.e., the first peak at $[-0.17 \mathrm{eV},-0.11 \mathrm{eV}]$ and the second 


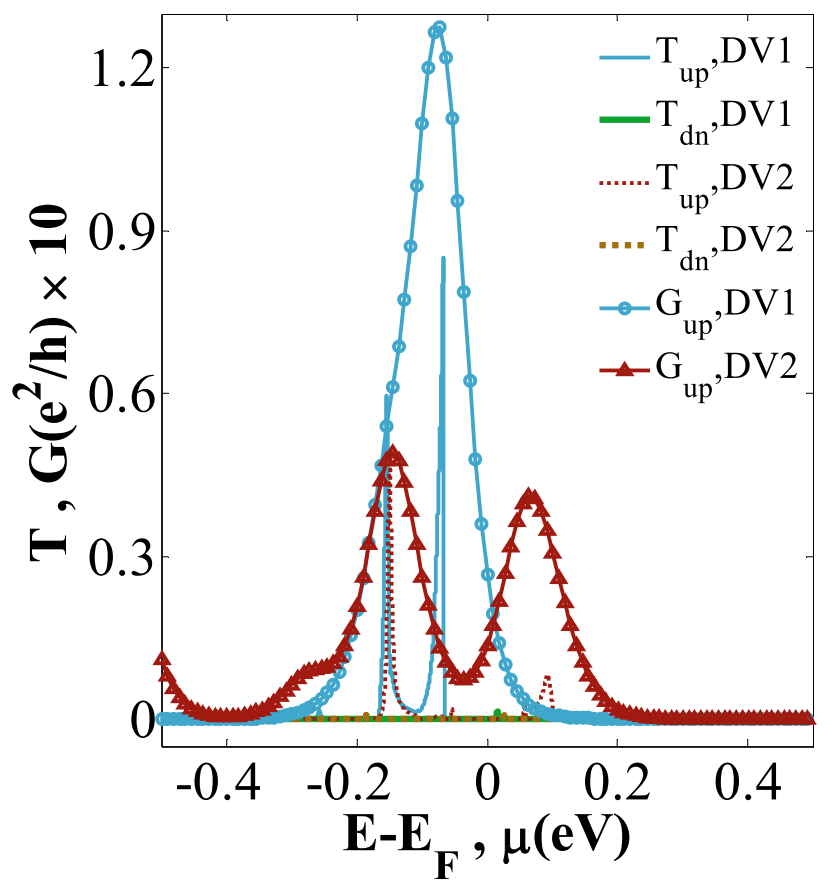

Figure 8. $T_{u p}$ and $T_{d n}$ channels versus $E-E_{\mathrm{F}}$ and $G_{u p}$ versus $\mu$ for the DV1and DV 2 configurations, at $T=300 \mathrm{~K}$, respectively.

one at $[0.07 \mathrm{eV}, 0.11 \mathrm{eV}]$ interval. This indicates that the current sign for the DV2 case is negative and changes to positive with the increase of $T_{\mathrm{R}}$ and NDTR. In DV1, the maximum values of $T_{u p}$ and $G_{u p}$ are almost obtained $30 \%$ and $90 \%$ larger than the stronger value in the DV2 (see Fig. 8). As a result, $I_{u p}$ in the DV2 case is negative and weaker. Nevertheless, the values of $T_{d n}$ and $G_{d n}$ are nearly zero in both cases and confirm the SFE. Accordingly, the spin-dependent transmission coefficient, the direction, and intensity of the current can be engineered using the DV orientation.

Figure 9 depicts the variation of $S_{u p}, S_{d n}, S_{s}$, and $S_{c}$ versus $\mu$ for the studied cases at $T=300 \mathrm{~K}$. By comparing $S_{S}$ values obtained with the DV1 and DV2 configurations, we find that the change of DV orientation may vary $S_{S}$. As shown in Fig. 9, the maximum value of $S_{S}$ is almost equal to $1.114 \mathrm{mV} / \mathrm{K}$ for the DV1 case, whereas, this value is $0.749 \mathrm{mV} / \mathrm{K}$ for the DV2 case. In the figure, $S_{s}$ color varies from orange to black, representing the contribution of the spin-down and spin-up electrons in $S_{s}$, respectively. The charge and spin thermoelectric efficiency are also assessed by calculating the electrical FOM $\left(Z_{e, c} T\right.$ and $\left.Z_{e, s} T\right)$ for each case study, and different $\mu$ values at room temperature (See insets in Fig. 9). The maximum values of $Z_{\mathrm{e}, c} T$ and $Z_{\mathrm{e}, s} T$ for the DV1 case are relatively $14 \%$ and $99 \%$ higher than those obtained by the DV2 case. This provides evidence that the thermoelectric efficiency of the case studies is controlled and enhanced by the DV.

\section{Conclusions}

In this research, the thermal SFE, spin-dependent electronic, and thermoelectric properties of the defected steplike GSNR junctions have numerically been studied. Each configuration was then subjected to the ferromagnetic exchange and local external electric fields. The divacancy effects were considered in the studied cases using the pentagon-octagon pentagon (5-8-5) type model. Higher amounts of spin-up current were also determined for high-temperature values, while the spin-down current is remained unchanged and equal to zero for all temperature values, when the right lead temperature $\left(T_{R}\right)$ increases. Hence, a satisfactory SFE and a spin polarization up to $99.99 \%$ were obtained in the studied GSNR junctions. The magnitude of the electric and ferromagnetic exchange fields is optimally selected to achieve the spin filtering effect with maximum efficiency. However, the SFE and current strength become more robust when the device size increases. This response behavior with localized transmission peaks could be achieved around the Fermi level if the divacancy was well oriented in the nanostructure. This provides evidence that the spin-dependent transmission coefficient, the direction, and intensity of the current can be engineered using the DV orientation. The NV and DV3 case studies showed insulator behavior, whereas the two other cases showed spin filtering behavior but with opposite spin current directions. It is realized that the DV1 case study (i.e., 47GE-14SE-23GE) can provide the strongest SFE and largest spin thermoelectric efficiency and enhance $Z_{e} T$. Some interesting transport properties such as the spin switching effect, rectifying behavior of the devices, and NDTR in thermal charge current were also observed. The maximum values of $\left|S_{s}\right|$ and the charge (spin) thermoelectric efficiencies for the 47GE-14SE-23GE case are obtained as $1.114 \mathrm{mV} / \mathrm{K}$ and 15.06 (101.4), respectively. These values are satisfactory and may be comparable to the values reported in other studies ${ }^{98,108,110}$. In general, the considered hybrid GSNRs can be used for thermoelectric applications using different system temperature sets. 


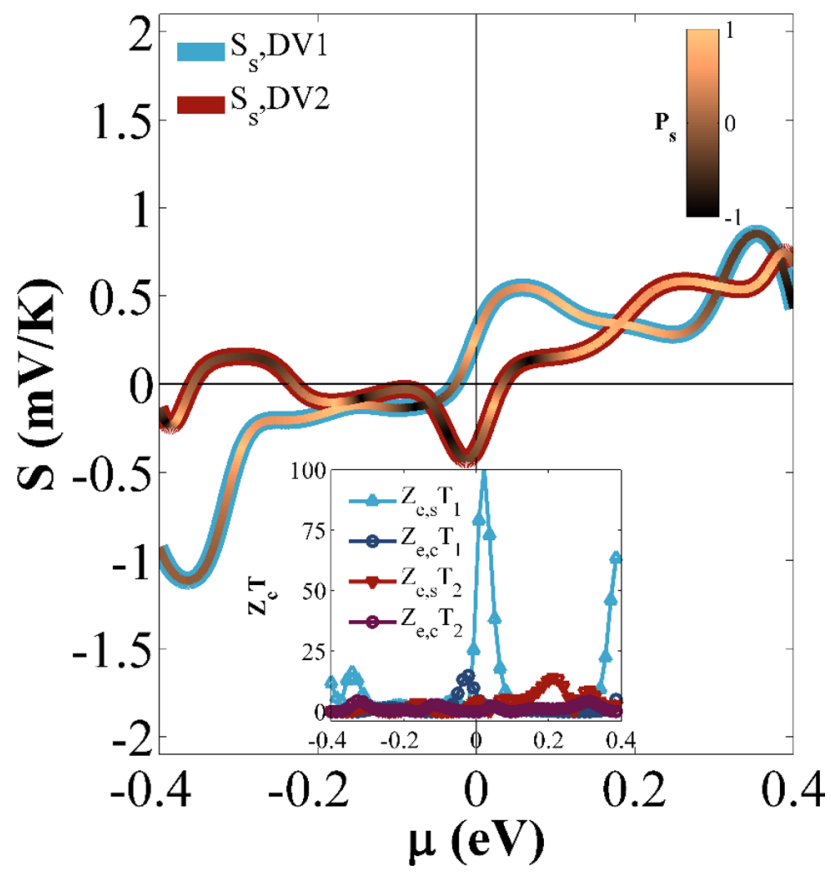

Figure 9. $S_{u p}, S_{d n}, S_{s}$, and $S_{c}$ against $\mu$ at room temperature for DV1and DV2 cases, respectively. $S_{S}$ color varies based on Seebeck polarization $\left(P_{s}\right)$ value. The inset shows $Z_{\mathrm{e}, s} T$ and $Z_{\mathrm{e}, c} T$ as a function of $\mu$.

Received: 17 March 2021; Accepted: 12 July 2021

Published online: 28 July 2021

\section{References}

1. Cowen, L. M., Atoyo, J., Carnie, M. J., Baran, D. \& Schroeder, B. C. Organic materials for thermoelectric energy generation. ECS J. Solid State Sci. Technol. 6, N3080 (2017).

2. Cui, C. et al. Bayesian optimization-based design of defect gamma-graphyne nanoribbons with high thermoelectric conversion efficiency. Carbon 176, 52-60 (2021).

3. Xiong, Y. et al. Chemically switchable n-type and p-type conduction in bismuth selenide nanoribbons for thermoelectric energy harvesting. ACS Nano 15, 2791-2799 (2021).

4. Zhang, Z., Xie, Y., Peng, Q. \& Chen, Y. A theoretical prediction of super high-performance thermoelectric materials based on $\mathrm{MoS}_{2} / \mathrm{WS}_{2}$ hybrid nanoribbons. Sci. Rep. 6, 1-8 (2016).

5. Bauer, G. E., Saitoh, E. \& Van Wees, B. J. Spin caloritronics. Nat. Mater. 11, 391-399 (2012).

6. Goennenwein, S. T. \& Bauer, G. E. Electron spins blow hot and cold. Nat. Nanotechnol. 7, 145-147 (2012).

7. Kuschel, T. \& Reiss, G. Charges ride the spin wave. Nat. Nanotechnol. 10, 22-24 (2015).

8. Uchida, K. et al. Observation of the spin Seebeck effect. Nature 455, 778-781 (2008).

9. Fu, H.-H., Wu, D.-D., Zhang, Z.-Q. \& Gu, L. Spin-dependent Seebeck effect, thermal colossal magnetoresistance and negative differential thermoelectric resistance in zigzag silicene nanoribbon heterojunciton. Sci. Rep. 5, 1-10 (2015).

10. Tan, X., Liu, L., Du, G.-F. \& Fu, H.-H. Thermal transport and spin-dependent Seebeck effect in parallel step-like zigzag graphene nanoribbon junctions. Phys. Chem. Chem. Phys. 22, 19100-19107 (2020).

11. Zhai, X. et al. Spin-valley caloritronics in silicene near room temperature. Phys. Rev. B 94, 245405 (2016).

12. Zou, F., Zhu, L., Gao, G., Wu, M. \& Yao, K. Temperature-controlled spin filter and spin valve based on Fe-doped monolayer $\mathrm{MoS}_{2}$. Phys. Chem. Chem. Phys. 18, 6053-6058 (2016).

13. Khoeini, F., Shakouri, K. \& Peeters, F. Peculiar half-metallic state in zigzag nanoribbons of $\mathrm{MoS}_{2}$ : Spin filtering. Phys. Rev. B 94, $125412(2016)$

14. Kim, W. Y. \& Kim, K. S. Prediction of very large values of magnetoresistance in a graphene nanoribbon device. Nat. Nanotechnol. 3, 408 (2008).

15. Wu, D.-D., Du, G.-F. \& Fu, H.-H. Spin-dependent Seebeck effect, and spin-filtering and diode effects in magnetic boron-nitrogen nanotube heterojunctions. J. Mater. Chem. C 8, 4486-4492 (2020).

16. Li, L., Partoens, B., Xu, W. \& Peeters, F. Electric-field modulation of linear dichroism and Faraday rotation in few-layer phosphorene. 2D Mater. 6, 015032 (2018).

17. Mortazavi, B., Dianat, A., Cuniberti, G. \& Rabczuk, T. Application of silicene, germanene and stanene for Na or Li ion storage: A theoretical investigation. Electrochim. Acta 213, 865-870 (2016).

18. Neek-Amal, M., Sadeghi, A., Berdiyorov, G. \& Peeters, F. Realization of free-standing silicene using bilayer graphene. Appl. Phys. Lett. 103, 261904 (2013).

19. Khoeini, F. \& Jafarkhani, Z. Tunable spin transport and quantum phase transitions in silicene materials and superlattices. J. Mater. Sci. 54, 14483-14494 (2019).

20. Li, L., Partoens, B. \& Peeters, F. Tuning the electronic properties of gated multilayer phosphorene: A self-consistent tight-binding study. Phys. Rev. B 97, 155424 (2018).

21. Anno, Y., Takei, K., Akita, S. \& Arie, T. Enhancing the thermoelectric device performance of graphene using isotopes and isotopic heterojunctions. Adv. Electron. Mater. 1, 1500175 (2015).

22. Khalkhali, M., Rajabpour, A. \& Khoeini, F. Thermal transport across grain boundaries in polycrystalline silicene: A multiscale modeling. Sci. Rep. 9, 1-12 (2019). 
23. Shirdel-Havar, M. \& Farghadan, R. Armchair graphene nanoribbons with giant spin thermoelectric efficiency. Phys. Chem. Chem. Phys. 20, 16853-16860 (2018).

24. Zberecki, K., Swirkowicz, R., Wierzbicki, M. \& Barnaś, J. Enhanced thermoelectric efficiency in ferromagnetic silicene nanoribbons terminated with hydrogen atoms. Phys. Chem. Chem. Phys. 16, 12900-12908 (2014).

25. Zong, P.-A. et al. Skutterudite with graphene-modified grain-boundary complexion enhances zT enabling high-efficiency thermoelectric device. Energy Environ. Sci. 10, 183-191 (2017).

26. Hicks, L. \& Dresselhaus, M. S. Effect of quantum-well structures on the thermoelectric figure of merit. Phys. Rev. B 47, 12727 (1993).

27. Sierra, J. F. et al. Thermoelectric spin voltage in graphene. Nat. Nanotechnol. 13, 107-111 (2018)

28. Balandin, A. A. et al. Superior thermal conductivity of single-layer graphene. Nano Lett. 8, 902-907 (2008).

29. Zberecki, K., Wierzbicki, M., Barnaś, J. \& Swirkowicz, R. Thermoelectric effects in silicene nanoribbons. Phys. Rev. B 88, 115404 (2013).

30. Yang, X. et al. Temperature-controlled giant thermal magnetoresistance behaviors in doped zigzag-edged silicene nanoribbons. RSC Adv. 4, 48539-48546 (2014).

31. Mahdavifar, M., Shekarforoush, S. \& Khoeini, F. Tunable electronic properties and electric-field-induced phase transition in phosphorene/graphene heterostructures. J. Phys. D Appl. Phys. 54, 095108 (2021).

32. Wang, N., Han, L., He, H., Park, N.-H. \& Koumoto, K. A novel high-performance photovoltaic-thermoelectric hybrid device. Energy Environ. Sci. 4, 3676-3679 (2011).

33. Parashchuk, T. et al. Insight into the transport properties and enhanced thermoelectric performance of $\mathrm{n}$-type $\mathrm{Pb} 1-\mathrm{xSbxTe} J$. Alloys Compd. 860, 158355 (2021).

34. Rostami, M., Ahmadi, I. \& Khoeini, F. Highly tunable charge transport in defective graphene nanoribbons under external local forces and constraints: A hybrid computational study. Results Phys. 20, 103770 (2021).

35. Yousefi, F., Khoeini, F. \& Rajabpour, A. Thermal conductivity and thermal rectification of nanoporous graphene: A molecular dynamics simulation. Int. J. Heat Mass Transf. 146, 118884 (2020).

36. Abdullah, N. R., Kareem, M. T., Rashid, H. O., Manolescu, A. \& Gudmundsson, V. Spin-polarised DFT modeling of electronic, magnetic, thermal and optical properties of silicene doped with transition metals. Phys. E Low-Dimens. Syst. Nanostruct. 129, $114644(2021)$

37. Tromer, R. M. et al. Electronic, optical and thermoelectric properties of boron-doped nitrogenated holey graphene. Phys. Chem. Chem. Phys. 22, 21147-21157 (2020).

38. Gholami, Z. \& Khoeini, F. Pure thermal spin current and perfect spin-filtering with negative differential thermoelectric resistance induced by proximity effect in graphene/silicene junctions. Sci. Rep. 11, 1-16 (2021).

39. Yang, K. et al. Enhanced thermoelectric properties in hybrid graphene/boron nitride nanoribbons. Phys. Rev. B 86, 045425 (2012).

40. Kiraly, B., Mannix, A. J., Hersam, M. C. \& Guisinger, N. P. Graphene-silicon heterostructures at the two-dimensional limit. Chem. Mater. 27, 6085-6090 (2015).

41. Kistanov, A. A., Cai, Y., Zhang, Y.-W., Dmitriev, S. V. \& Zhou, K. Strain and water effects on the electronic structure and chemical activity of in-plane graphene/silicene heterostructure. J. Phys. Condens. Matter 29, 095302 (2017).

42. Li, G. et al. Stable silicene in graphene/silicene Van der Waals heterostructures. Adv. Mater. 30, 1804650 (2018).

43. Liu, B. et al. Interface thermal conductance and rectification in hybrid graphene/silicene monolayer. Carbon 79, 236-244 (2014).

44. Drissi, L., Saidi, E., Bousmina, M. \& Fassi-Fehri, O. DFT investigations of the hydrogenation effect on silicene/graphene hybrids. J. Phys. Condens. Matter 24, 485502 (2012).

45. Feng, Y. et al. Multiple synergistic effects of graphene-based hybrid and hexagonal born nitride in enhancing thermal conductivity and flame retardancy of epoxy. Chem. Eng. J. 379, 122402 (2020).

46. Liu, B. et al. Interfacial thermal conductance of a silicene/graphene bilayer heterostructure and the effect of hydrogenation. ACS Appl. Mater. Interfaces 6, 18180-18188 (2014).

47. Ren, J. et al. Enhanced thermal conductivity of epoxy composites by introducing graphene@ boron nitride nanosheets hybrid nanoparticles. Mater. Des. 191, 108663 (2020).

48. Chang, P.-H. \& Nikolić, B. K. Edge currents and nanopore arrays in zigzag and chiral graphene nanoribbons as a route toward high-Z T thermoelectrics. Phys. Rev. B 86, 041406 (2012).

49. Gunst, T., Markussen, T., Jauho, A.-P. \& Brandbyge, M. Thermoelectric properties of finite graphene antidot lattices. Phys. Rev. $B$ 84, 155449 (2011).

50. Liu, Q.-B., Wu, D.-D. \& Fu, H.-H. Edge-defect induced spin-dependent Seebeck effect and spin figure of merit in graphene nanoribbons. Phys. Chem. Chem. Phys. 19, 27132-27139 (2017).

51. Oh, J. et al. Significantly reduced thermal conductivity and enhanced thermoelectric properties of single-and bi-layer graphene nanomeshes with sub-10 nm neck-width. Nano Energy 35, 26-35 (2017).

52. Yu, D., Lupton, E. M., Gao, H., Zhang, C. \& Liu, F. A unified geometric rule for designing nanomagnetism in graphene. Nano Res. 1, 497-501 (2008).

53. Chen, X., Liu, Y., Gu, B.-L., Duan, W. \& Liu, F. Giant room-temperature spin caloritronics in spin-semiconducting graphene nanoribbons. Phys. Rev. B 90, 121403 (2014).

54. Chico, L., Orellana, P., Rosales, L. \& Pacheco, M. Spin and charge caloritronics in bilayer graphene flakes with magnetic contacts. Phys. Rev. Appl. 8, 054029 (2017).

55. Zhang, Z.-Y. \& Guo, W. Cutting monolayer graphene into flexible spin filters. Carbon 115, 43-49 (2017).

56. Sonvane, Y., Gupta, S. K., Raval, P., Lukačević, I. \& Thakor, P. B. Length, width and roughness dependent thermal conductivity of graphene nanoribbons. Chem. Phys. Lett. 634, 16-19 (2015).

57. Ju, S. \& Liang, X. Thermal rectification and phonon scattering in asymmetric silicon nanoribbons. J. Appl. Phys. 112, 024307 (2012).

58. Khoeini, F. \& Shokri, A. A. Electronic transport in Bi-asymmetric T-shaped graphene nanoribbons. J. Comput. Theor. Nanosci. 8, 740-745 (2011).

59. Yang, N., Zhang, G. \& Li, B. Thermal rectification in asymmetric graphene ribbons. Appl. Phys. Lett. 95, 033107 (2009).

60. Zhang, Z., Wu, Z., Chang, K. \& Peeters, F. Resonant tunneling through S-and U-shaped graphene nanoribbons. Nanotechnology 20, 415203 (2009).

61. Khoeini, F. \& Shokri, A. Modeling of transport in a glider-like composite of GNR/CNT/GNR junctions. J. Comput. Theor. Nanosci. 8, 1315-1320 (2011).

62. Rojo, M. M. et al. Thermal transport across graphene step junctions. 2D Mater. 6, 011005 (2018).

63. Chen, H. \& Stoddart, J. F. From molecular to supramolecular electronics. Nat. Rev. Mater. 1-25 (2021).

64. Chen, L. et al. Oriented graphene nanoribbons embedded in hexagonal boron nitride trenches. Nat. Commun. 8, 1-6 (2017).

65. Fan, T. et al. Controllable size-selective method to prepare graphene quantum dots from graphene oxide. Nanoscale Res. Lett. 10, 1-8 (2015).

66. Kim, Y., Jeong, W., Kim, K., Lee, W. \& Reddy, P. Electrostatic control of thermoelectricity in molecular junctions. Nat. Nanotechnol. 9, 881 (2014). 
67. Llinas, J. P. et al. Short-channel field-effect transistors with 9-atom and 13-atom wide graphene nanoribbons. Nat. Commun. 8, 1-6 (2017).

68. Wang, H. S. et al. Towards chirality control of graphene nanoribbons embedded in hexagonal boron nitride. Nat. Mater. 20, 202-207 (2021).

69. Wang, X. et al. Room-temperature all-semiconducting sub-10-nm graphene nanoribbon field-effect transistors. Phys. Rev. Lett. 100, 206803 (2008).

70. Reddy, P., Jang, S.-Y., Segalman, R. A. \& Majumdar, A. Thermoelectricity in molecular junctions. Science 315, 1568-1571 (2007).

71. Widawsky, J. R. et al. Length-dependent thermopower of highly conducting Au-C bonded single molecule junctions. Nano Lett. 13, 2889-2894 (2013).

72. Unsal, E., Senger, R. \& Sevinçli, H. Enhancement of thermoelectric efficiency of T-HfSe $e_{2}$ via nanostructuring. Phys. Rev. B 103, 014104 (2021).

73. Ni, Y. et al. Spin seebeck effect and thermal colossal magnetoresistance in graphene nanoribbon heterojunction. Sci. Rep. 3, 1-5 (2013).

74. Huang, H., Zheng, A., Gao, G. \& Yao, K. Thermal spin filtering effect and giant magnetoresistance of half-metallic graphene nanoribbon co-doped with non-metallic Nitrogen and Boron. J. Magn. Magn. Mater. 449, 522-529 (2018).

75. Liu, Y. et al. Multi-functional spintronic devices based on boron-or aluminum-doped silicene nanoribbons. Nanotechnology 29, 125201 (2018).

76. Zeng, M., Huang, W. \& Liang, G. Spin-dependent thermoelectric effects in graphene-based spin valves. Nanoscale 5, 200-208 (2013).

77. Matthes, L., Hannewald, K. \& Bechstedt, F. Ab initio investigation of graphene-based one-dimensional superlattices and their interfaces. Phys. Rev. B 86, 205409 (2012).

78. An, R.-L. et al. Vacancy effects on electric and thermoelectric properties of zigzag silicene nanoribbons. J. Phys. Chem. C 118, 21339-21346 (2014).

79. Kim, Y., Ihm, J., Yoon, E. \& Lee, G.-D. Dynamics and stability of divacancy defects in graphene. Phys. Rev. B 84, 075445 (2011).

80. Jippo, H., Ohfuchi, M. \& Kaneta, C. Theoretical study on electron transport properties of graphene sheets with two-and onedimensional periodic nanoholes. Phys. Rev. B 84, 075467 (2011).

81. Lieb, E. H. Two theorems on the Hubbard model. Phys. Rev. Lett. 62, 1201 (1989).

82. Datta, S. Quantum Transport: Atom to Transistor (Cambridge University PRESS, 2005).

83. Büttiker, M., Imry, Y., Landauer, R. \& Pinhas, S. Generalized many-channel conductance formula with application to small rings. Phys. Rev. B 31, 6207 (1985).

84. Chen, A.-B., Wang, X.-F., Vasilopoulos, P., Zhai, M.-X. \& Liu, Y.-S. Spin-dependent ballistic transport properties and electronic structures of pristine and edge-doped zigzag silicene nanoribbons: Large magnetoresistance. Phys. Chem. Chem. Phys. 16, 5113-5118 (2014).

85. Gunlycke, D., Lawler, H. \& White, C. Room-temperature ballistic transport in narrow graphene strips. Phys. Rev. B 75, 085418 (2007).

86. Kaneko, S., Tsuchiya, H., Kamakura, Y., Mori, N. \& Ogawa, M. Theoretical performance estimation of silicene, germanene, and graphene nanoribbon field-effect transistors under ballistic transport. Appl. Phys. Express 7, 035102 (2014).

87. Vogt, P. \& Le Lay, G. Silicene: Prediction, Synthesis, Application (Springer, 2018).

88. Liu, C.-C., Feng, W. \& Yao, Y. Quantum spin Hall effect in silicene and two-dimensional germanium. Phys. Rev. Lett. 107, 076802 (2011).

89. Mahdavifar, M. \& Khoeini, F. Highly tunable charge and spin transport in silicene junctions: phase transitions and half-metallic states. Nanotechnology 29, 325203 (2018).

90. Reich, S., Maultzsch, J., Thomsen, C. \& Ordejon, P. Tight-binding description of graphene. Phys. Rev. B 66, 035412 (2002).

91. Yokoyama, T. Controllable valley and spin transport in ferromagnetic silicene junctions. Phys. Rev. B 87, 241409 (2013).

92. Barrios-Vargas, J. E. et al. Electrical and thermal transport in coplanar polycrystalline graphene-hbn heterostructures. Nano Lett. 17, 1660-1664 (2017)

93. Qasemnazhand, M., Khoeini, F. \& Shekarforoush, S. Electronic transport properties in the stable phase of a cumulene/ $\mathrm{B}_{7} / \mathrm{cumu}^{-}$ lene molecular bridge investigated using density functional theory and a tight-binding method. New J. Chem. 43, 16515-16523 (2019).

94. Khoeini, F., Khoeini, F. \& Shokri, A. Peculiar transport properties in Z-shaped graphene nanoribbons: A nanoscale NOR gate. Thin Solid Films 548, 443-448 (2013).

95. Sivan, U. \& Imry, Y. Multichannel Landauer formula for thermoelectric transport with application to thermopower near the mobility edge. Phys. Rev. B 33, 551 (1986).

96. Wu, D. et al. Pure spin current generated in thermally driven molecular magnetic junctions: A promising mechanism for thermoelectric conversion. J. Mater. Chem. A 7, 19037-19044 (2019).

97. Li, J., Wang, B., Xu, F., Wei, Y. \& Wang, J. Spin-dependent Seebeck effects in graphene-based molecular junctions. Phys. Rev. B 93, $195426(2016)$

98. Wierzbicki, M., Swirkowicz, R. \& Barnaś, J. Giant spin thermoelectric efficiency in ferromagnetic graphene nanoribbons with antidots. Phys. Rev. B 88, 235434 (2013).

99. Liu, J. et al. N-type organic thermoelectrics: demonstration of ZT >0.3. Nat. Commun. 11, 1-9 (2020).

100. Mubarak, A. The mechanical, optical and thermoelectric properties of $\mathrm{MCoF}_{3}(\mathrm{M}=\mathrm{K}$ and $\mathrm{Rb})$ compounds. Mod. Phys. Lett. $B$ 31, 1750033 (2017).

101. Le, P., Davoudiniya, M., Mirabbaszadeh, K., Hoi, B. \& Yarmohammadi, M. Combined electric and magnetic field-induced anisotropic tunable electronic phase transition in AB-stacked bilayer phosphorene. Physica E 106, 250-257 (2019).

102. Tran, V.-T., Saint-Martin, J. \& Dollfus, P. Large on/off current ratio in hybrid graphene/BN nanoribbons by transverse electric field-induced control of bandgap. Appl. Phys. Lett. 105, 073114 (2014).

103. Liu, Y.-S., Wang, X.-F. \& Chi, F. Non-magnetic doping induced a high spin-filter efficiency and large spin Seebeck effect in zigzag graphene nanoribbons. J. Mater. Chem. C 1, 8046-8051 (2013).

104. Pal, A. N. et al. Nonmagnetic single-molecule spin-filter based on quantum interference. Nat. Commun. 10, 1-8 (2019).

105. Paudel, T. R. \& Tsymbal, E. Y. Spin filtering in $\mathrm{CrI}_{3}$ tunnel junctions. ACS Appl. Mater. Interfaces 11, 15781-15787 (2019).

106. Roch, J. G. et al. Spin-polarized electrons in monolayer $\mathrm{MoS}_{2}$. Nat. Nanotechnol. 14, 432-436 (2019).

107. Tsai, W.-F. et al. Gated silicene as a tunable source of nearly 100\% spin-polarized electrons. Nat. Commun. 4, 1-6 (2013).

108. Yang, X.-F., Liu, Y.-S., Feng, J.-F. \& Wang, X.-F. Large spin Seebeck effects in zigzag-edge silicene nanoribbons. AIP Adv. 4, $087116(2014)$

109. Zhai, M.-X. et al. Giant magnetoresistance and spin Seebeck coefficient in zigzag a-graphyne nanoribbons. Nanoscale 6, 1112111129 (2014).

110. Sadeghi, H., Sangtarash, S. \& Lambert, C. J. Enhancing the thermoelectric figure of merit in engineered graphene nanoribbons. Beilstein J. Nanotechnol. 6, 1176-1182 (2015).

111. Palacios, J. J., Fernández-Rossier, J. \& Brey, L. Vacancy-induced magnetism in graphene and graphene ribbons. Phys. Rev. B 77, $195428(2008)$ 


\section{Author contributions}

Z.G.: writing, analysis. F.K.: Supervision, writing, verification.

\section{Competing interests}

The authors declare no competing interests.

\section{Additional information}

Correspondence and requests for materials should be addressed to F.K.

Reprints and permissions information is available at www.nature.com/reprints.

Publisher's note Springer Nature remains neutral with regard to jurisdictional claims in published maps and institutional affiliations.

(c) (i) Open Access This article is licensed under a Creative Commons Attribution 4.0 International License, which permits use, sharing, adaptation, distribution and reproduction in any medium or format, as long as you give appropriate credit to the original author(s) and the source, provide a link to the Creative Commons licence, and indicate if changes were made. The images or other third party material in this article are included in the article's Creative Commons licence, unless indicated otherwise in a credit line to the material. If material is not included in the article's Creative Commons licence and your intended use is not permitted by statutory regulation or exceeds the permitted use, you will need to obtain permission directly from the copyright holder. To view a copy of this licence, visit http://creativecommons.org/licenses/by/4.0/.

(C) The Author(s) 2021 